Research Article

\title{
An Orderly EV Charging Scheduling Method Based on Deep Learning in Cloud-Edge Collaborative Environment
}

\author{
Jiayong Zhong (iD) and Xiaofu Xiong \\ State Key Laboratory of Power Transmission Equipment \& System Security and New Technology, Chongqing University, \\ Chongqing, China
}

Correspondence should be addressed to Jiayong Zhong; 20071102080@cqu.edu.cn

Received 1 November 2020; Revised 10 December 2020; Accepted 24 December 2020; Published 8 January 2021

Academic Editor: Yi-Zhang Jiang

Copyright ( $) 2021$ Jiayong Zhong and Xiaofu Xiong. This is an open access article distributed under the Creative Commons Attribution License, which permits unrestricted use, distribution, and reproduction in any medium, provided the original work is properly cited.

\begin{abstract}
The rapid increase of the number of electric vehicles (EVs) has posed great challenges to the safe operation of the distribution network. Therefore, this paper proposes an ordered charging scheduling method for EV in the cloud-edge collaborative environment. Firstly, the uncertainty of user load demands, charging station requirements, and renewable outputs are taken into consideration. Correspondingly, the residential distribution points, EV charging stations, and renewable plants are regarded as the edge nodes. Then, the load demands and renewable outputs are predicted by a model combined with the convolutional neural network and deep belief network (CNN-DBN). Secondly, the power supply plans for charging stations are determined at the cloud side aiming at minimizing the operating cost of the distribution network via collecting the forecasting results. Finally, the charging station formulates the personalized charging scheduling strategies according to EV's charging plans and the charging demands in order to follow the supply plan. The simulation results show that the load peak-to-valley difference and standard deviation of the proposed algorithm are reduced by $30.13 \%$ and $16.94 \%$, respectively, compared with the disorderly charging and discharging behavior, which has verified the effectiveness and feasibility of the proposed method.
\end{abstract}

\section{Introduction}

With the popularization of electric vehicles (EVs), the rapid increase of charging demands has posed great challenges to the safe operation of the distribution networks [1]. For centralized charging stations, due to the concentrated charging characteristic of EVs, the impact of disorderly charging behavior on the grid cannot be ignored, especially in case that the number of charging users is large. Specifically, as the charging behavior of EVs is highly correlated with user's travel habits, thus disorderly charging behavior may cause the expansion of the peak-to-valley gap in the distribution network and lead to a series of problems such as active/reactive power supply and demand tensions, frequency/voltage deviations, and network losses increase [2-4]. Therefore, how to guide the orderly charging process of EVs and reduce the influence of charging behavior uncertainty on the distribution network operation has received extensive attention in recent years.
According to $[5,6]$, the number of EVs that need to be charged in a day shows obvious peak-to-valley characteristics. For example, EV charging demand is usually low during the morning peak, while it will increase after people getting off work. Therefore, the distribution network must take into account the charging demands of charging stations, residential power load demands, and the output of distributed renewable sources when determining the powers allocated to the charging stations in different time periods. Then, the economic operation of the distribution network can be realized without violating the safe operation constraints of the distribution network [7-9].

However, in the existing related works, the forecasting of charging demands, residential loads, and renewable outputs is often conducted by using the time series analysis methods or artificial intelligence methods in the control center of distribution network at cloud side. Therefore, the computational burden at the cloud-side control center is usually heavy. 
With the rapid development of edge computing technology, the perception breadth and depth of the distribution network have been further enhanced. Specifically, it is feasible to set up edge computing devices at the residential distribution stations, charging stations, and renewable plants to provide more accurate forecasting capabilities. Correspondingly, the control center at the cloud side can formulate a more economical and friendly charging plan based on the predicted results and thereby improve the operation reliability of the distribution network.

Therefore, this paper proposes an orderly charging scheduling scheme for EVs based on cloud-side collaborative architecture. The main contributions are summarized as follows:

(1) A forecasting model based on convolutional neural network and deep belief network to predict residential loads, charging demands, and renewable outputs is proposed by installing edge computing devices in residential areas, charging stations, and renewable plants in the distribution network. Under the proposed scheme, the power forecasting result of each edge computing node is uploaded to the cloudside control center in distribution network to formulate the orderly charging scheduling strategy for EVs.

(2) A two-stage EV charging scheduling strategy is proposed according to the predicted power results. Firstly, the cloud-side control center in distribution network formulates the overall charging plan for EV charging stations based on the collected power forecast results aiming at minimizing the operating cost of the distribution network. Secondly, each EV charging station formulates a charging scheduling strategy for each EV according to the planned curve.

The rest of this paper is organized as follows. Related work is analyzed in Section 2. The overall power forecasting framework based on cloud-edge collaborative environment is given in Section 3. In Section 4, the proposed CNN-DBN forecasting method is discussed. Orderly charging scheduling strategy for EVs is given in Section 5. Simulations and results are discussed in Section 6. Finally, conclusion is given in Section 7.

\section{Related Work}

2.1. Load Forecasting Methods. The load forecasting results are the basis for determining the capacity of distribution network equipment and formulating the corresponding operating conditions (e.g., transformer ratio, generator output, shunt capacitor capacity, and EV charging plan). Therefore, increasing the accuracy of load forecasting is of great significance to improve the reliability and economy of the distribution network. The existing load forecasting methods can be roughly divided into traditional nonartificial intelligence methods, machine learning methods, and artificial intelligence methods.

\subsubsection{Traditional Forecasting Methods Based on Nonartificial} Intelligence Technology. Nonartificial intelligence methods are generally based on the time-characteristics of the load demands. Then, statistical methods are usually used to realize the long-time load demand forecasting. For example, in [10], a short-term load forecasting method based on the linear regression model is proposed. However, the significant disadvantage of linear regression analysis is that the outliers have great impacts on the forecasting accuracy. In [11], a method based on Markov model and likelihood ratio test is proposed. However, a drawback of the method in [11] is the fact that it is very sensitive to the time span. In [12], a power load forecasting model based on the combination of finite Fourier series and time series analysis theory is proposed. The forecasting accuracy is very sensitive to the number of Fourier series used for load curve fitting. In addition, with the large-scale integration of EVs and renewable power generation equipment on the load side, the nonlinear characteristics, time-varying nature, and uncertainties of power load changes become more and more obvious. Considering the poor forecasting accuracy and robustness of traditional methods, it is difficult to establish a suitable mathematical model to clearly express the relationships among the forecasting results and influencing variables.

2.1.2. Forecasting Methods Based on Artificial Intelligence Technology. In recent years, the data-driven, model-free artificial intelligence algorithms have shown great development prospects in the power forecasting field. Random forest $[13,14]$, BP neural network [15], support vector machine [16], long short-term memory network [17], and convolutional neural network [18] have been used in the power forecasting by the researchers. For example, in [19], a short-term load forecasting method based on the combination of fuzzy time series (FTS) and convolutional neural network (CNN) is proposed. However, traditional CNN cannot accurately capture the timing information contained in the time series and also has long training time. In [20], a load forecasting method with long short-term memory network and genetic algorithm is proposed. The input data is selected as factors that affect the load demands while the output is the load curve. However, the long short-term memory network adds complex gate elements (i.e., forget gate, input gate, candidate gate, and output gate) to each neural unit in the hidden layers. Therefore, the training efficiency of LSTM may be low. In [21], a SVM method based on gray wolf optimization is proposed to forecast the load demands. However, SVM method needs continuous data samples, and its classification performance may not be satisfactory when the training data size is too large. 
2.2. Orderly Charging Scheduling Strategies. In all the scheduling strategies, based on the predicted results of the load demands in different periods of the next day, the benchmark operation point of the regulation equipment and the expected charging plan of EVs are determined in order to optimize the operation cost of the distribution network and maximize users' benefits. For example, in [22], a day-ahead scheduling scheme considering the uncertainties of residential loads and EV charging behaviors is proposed. Besides, a multiobjective optimization model considering feeder reconfiguration and shunt capacitor switching is established to reduce the operating cost of the distribution network, active power losses, and greenhouse gas emissions. In order to reduce the impacts of plug-in hybrid EVs (PHEV), distributed battery energy storage systems (DBESS), and distributed generators (DGs) in the power distribution system, a 24 -hour power scheduling plan is proposed in [23]. In [24], a two-stage optimization algorithm to design the EV charging plan is proposed, comprehensively considering the interests of EV users and distribution network operators. In [25], the uncertainty of EV charging station load is taken into account, and a microgrid day-ahead scheduling optimization scheme based on Monte Carlo simulation is proposed. In [26], a robust scheduling strategy considering the uncertainties of the realtime charging price and EV user charging demand is proposed. In [27], an optimization model for microgrid operation considering the EV charging and energy storage integration stations is proposed. The model predictive control (MPC) method is used to formulate the charging schedule with the goal of minimizing the total daily operating cost. Similarly, [28, 29] also proposed an orderly charging scheduling scheme for EVs based on MPC. However, the disadvantage of the MPC is that real-time rolling optimization requires the additional computational consumptions. When there are too many constraints and decision variables, it may be impossible to find a suitable scheduling strategy within a limited time. In addition, most of the existing optimal charging scheduling strategies for EVs are solved in the control center of the distribution network with a centralized way. When the scale of EVs is too large, it is difficult to determine the charging plan for each EV. To address the problem, a feasible method is proposed based on the hierarchical idea. Specifically, the distribution network sets the charging plan of each charging station while each charging station independently determines the charging period and charging power of each EV.

Based on the above analysis, this paper proposes an orderly charging scheduling method for EVs based on cloud-edge collaboration and deep learning technology. Firstly, the residential distribution areas, charging stations, and renewable plants are regarded as edge computing nodes which are used to forecast the residential loads, charging demands, and renewable outputs, respectively. Secondly, the control center in distribution network at cloud side proposes the charging plan for each charging station based on the predicted results to minimize the operation cost of the distribution network. Finally, each charging station sets a personalized charging plan for each EV based on the user's charging demand.

\section{Orderly EV Charging Strategy in Cloud-Edge Collaborative Environment}

As shown in Figure 1, the distribution network usually contains residential loads, charging stations, renewable plants, smart breakers, communication facilities, etc. With the rapid spread of electric vehicles, disorderly charging behaviors will bring serious impact on the safety of distribution network. Therefore, it is necessary to reasonably arrange the charging plan of charging station on the basis of charging load forecasting results. Different from the traditional centralized load forecasting and scheduling schemes which have a drawback of huge computing burden at the cloud-side control center, in this paper, the proposed electric vehicle charging scheduling strategy based on cloud-edge collaboration includes the following three parts.

3.1. Power Forecasting at Edge Sides. The residential distribution areas, centralized charging stations, and renewable plants in the distribution network are equipped with edge computing nodes with the capacities of data storage, calculation, and analysis. The corresponding residential loads, charging demands, and renewable outputs are predicted at the edge sides and then uploaded to the cloud-side control center.

\subsection{Power Supply Plan for Charging Stations Determined at} Cloud Side. The control center in distribution network at cloud side draws up the charging plans for the EV charging stations aiming at minimizing the distribution network operation cost including power purchase costs and renewable power generation revenue. In other words, based on the forecasting results, the charging plan (i.e., power supply plan) of each charging station can be obtained.

3.3. Orderly Charging Scheduling Strategy for EVs. Each charging station provides a personalized charging plan for each EV based on the remaining SoC and expected charging time, with the goal of minimizing the implementation of the charging plan. Specifically, the charging power and charging period of each EV are determined.

\section{Power Forecasting Based on the CNN-DBN}

Because that there are many factors which affect the residential loads, EV charging demands and renewable outputs such as season, workday/rest days, geographical factors, etc., it has the disadvantages as low training efficiency and poor forecasting accuracy to directly take so many influencing factors as input variables and extract high-dimensional features for power forecasting in traditional learning-based methods. Therefore, this paper adopts the power forecasting model based on CNN and DBN as shown in Figure 2. 


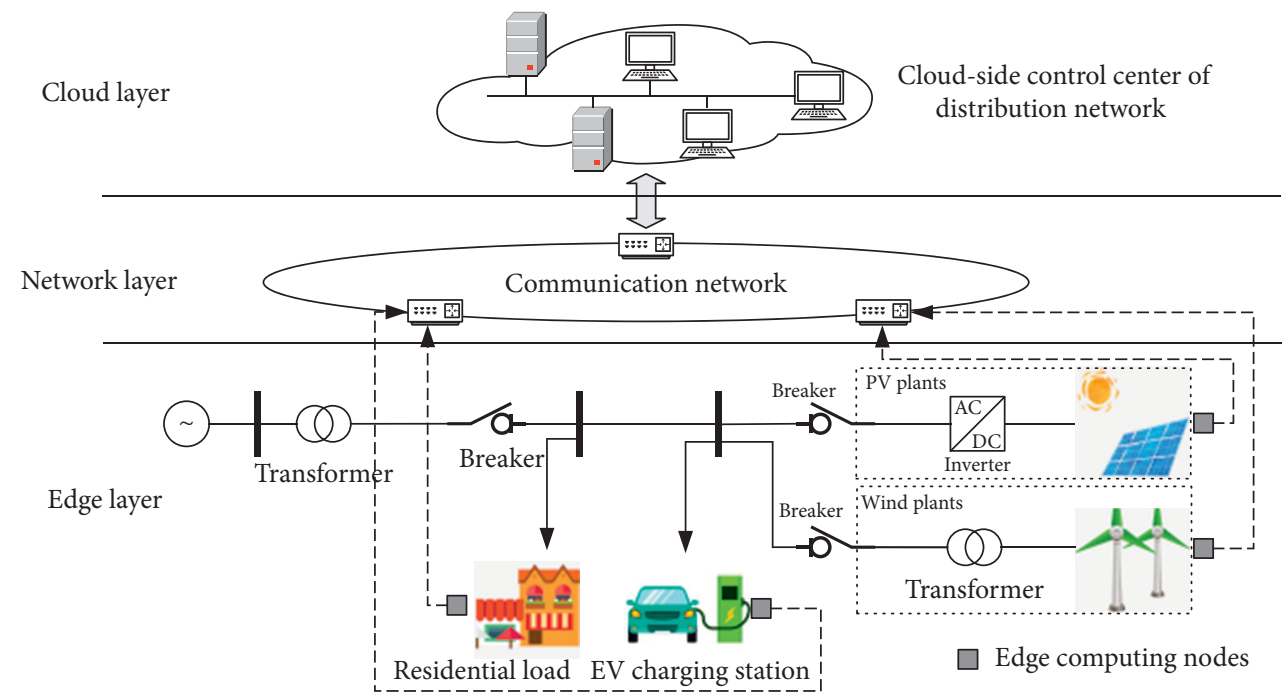

FIGURE 1: EV orderly charging scheduling strategy in cloud-edge collaborative environment.

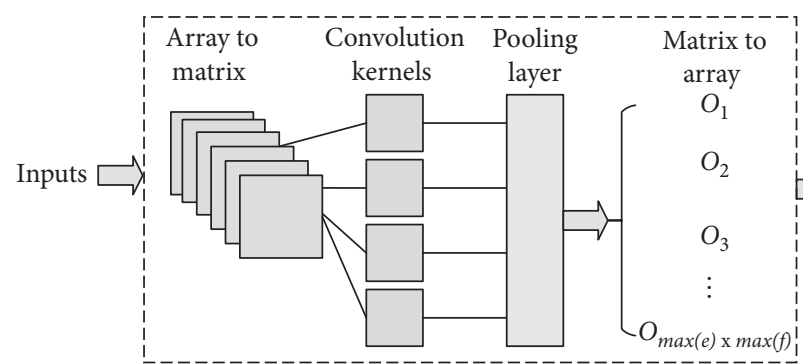

CNN

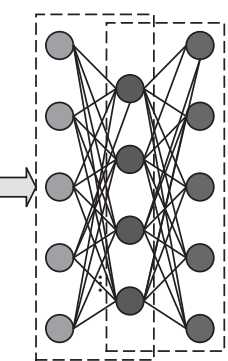

RBM1 RBM2

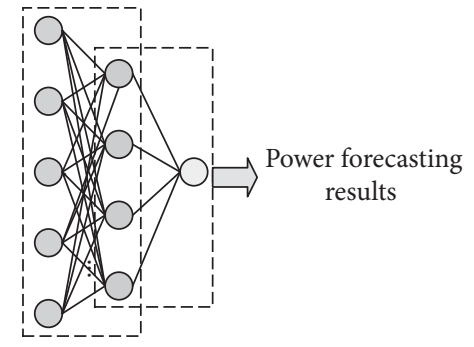

$\mathrm{RBM}_{I} \mathrm{BPNN}$

FIGURE 2: DBN-based power forecasting model.

Specifically, CNN is used to extract the hidden features of high-dimensional input data to construct a new data model for power forecasting. DBN with a layer of back-propagation neural network (BPNN) is used to establish a regression model between the extracted data features and the power forecasting results. It should be pointed out that CNN-DBN-based models deployed in the edge computing nodes installed in residential distribution areas, centralized charging stations, and renewable plants adopt the same type of input variables and network structure. The differences among the CNN-DBN models are the weighting coefficients and convolutions kernels obtained from the training processes.

4.1. CNN for Feature Extraction of Input Data. The structures before full-connection layer of $\mathrm{CNN}$, i.e., the convolution and pooling layers, are used to extract the hidden features of high-dimensional input data. The inputs of convolution layer are the original data while the corresponding outputs are the results which are convolved with the convolution kernels. Let $K_{A B}(A, B \in \mathrm{N})$ be the convolution kernel matrix and $X=\left[X_{1}, X_{2}, \ldots, X_{C}\right], C \in \mathrm{N}$, represent the input data vector. Transfer $X$ into a matrix with $D \times E$ dimensions, denoted as $X_{D E}$. Then, the relationships among the inputs and outputs in convolution layer satisfy

$$
S(e, f)=\sum_{A} \sum_{B} X(i+A, j+B) K_{A B},
$$

where $S(e, f)$ is the element of output matrix in convolution layer.

Furtherly, to avoid over fitting and reduce the number of hidden features of input data, the maximum pooling technology is used in $\mathrm{CNN}$ pooling layer to process the outputs of convolution layer. The output of pooling layer is shown as follows:

$$
O(e, f)=\operatorname{Max}(S(e, f)),
$$

where $O(e, f)$ are the matrix element after pooling operation while the dimensions of the matrix $O$ satisfy

$$
\begin{aligned}
& \max (e)=\left\lceil\frac{C}{B}\right\rceil, \\
& \max (f)=\left\lceil\frac{D}{A}\right\rceil,
\end{aligned}
$$

where $\lceil\cdot\rceil$ is the up-rounding operator.

Finally, considering that $\mathrm{CNN}$ is used to extract the hidden features and construct a new data model for the DBN, the final outputs of pooling layer should be converted 
into a 1-dimensional vector with $\max (e) \times \max (f)$ elements, denoted as $\left\{O_{1}, O_{2}, \ldots, O_{\max (e) \times \max (f)}\right\}$.

4.2. $D B N$ for Power Forecasting. As shown in Figure 2, the DBN is composed of multiple restricted Boltzmann machines (RBMs). For any RBM, there are two layers. The first layer is the visible layer (denoted as $V$ ) which is used to receive external input variables such as the outputs of $\mathrm{CNN}$ or the outputs from the previous RBM. The second layer is the hidden layer (denoted as $H$ ), which is used to extract the feature information contained in the input data. Suppose the input and output components of the $i$ th $(i=1,2, \ldots, I)$ RBM are $V_{i}=\left(v_{1}^{i}, v_{2}^{i}, \ldots, v_{m}^{i}\right)$ and $H_{i}=\left(h_{1}^{i}, h_{2}^{i}, \ldots, h_{m}^{i}\right)$. A fully connected structure with weight coefficients is used between the visible and hidden layers while the units on the same layer are not connected to each other. Let the weight coefficients between the visible layer and the hidden layer be $w_{i}=\left\{w_{p q}^{i}\right\}(p=1,2, \ldots, m ; q=1,2, \ldots, n)$. Therefore, the $i$ th RBM can be described as $\theta_{i}=\left\{V_{i}, H_{i}, w_{i}\right\}$.

To evaluate the working status of RBM, the following energy function is used:

$$
E\left(V_{i}, H_{i} \mid \theta_{i}\right)=-\sum_{p=1}^{m} a_{p}^{i} v_{p}^{i}-\sum_{q=1}^{n} b_{q}^{j} h_{q}^{j}-\sum_{p=1}^{m} \sum_{q=1}^{n} w_{p q}^{i} v_{p}^{i} h_{q}^{j},
$$

where ai $p$ and $b i q$ are the activation states of the $p$ th visible unit and the $q$ th hidden unit, which are represented by variables from 0 to 1 . Since the units in the same layer are not connected to each other, the joint probability distribution of visible nodes and hidden nodes is

$$
\left\{\begin{array}{l}
P\left(V_{i}, H_{i} \mid \theta_{i}\right)=\frac{e^{-E\left(V_{i}, H_{i} \mid \theta_{i}\right)}}{Z\left(\theta_{i}\right)}, \\
Z\left(\theta_{i}\right)=\sum_{V_{i}, H_{i}} e^{-E\left(V_{i}, H_{i} \mid \theta_{i}\right)},
\end{array}\right.
$$

where $Z\left(\theta_{i}\right)$ is the normalization factor, which is the energy function of all possible states of the $i$ th RBM. In addition, the probabilities of the $p$ th visible unit and the $q$ th hidden unit can be given as

$$
\begin{aligned}
& P\left(a_{p}^{i} \mid H_{i}\right)=\sigma\left(h_{q}^{j}+\sum_{q=1}^{n} b_{q}^{i} w_{p q}^{i}\right), \\
& P\left(b_{q}^{i} \mid V_{i}\right)=\sigma\left(v_{q}^{j}+\sum_{p=1}^{m} a_{p}^{i} w_{p q}^{i}\right),
\end{aligned}
$$

where $\sigma(x)$ is the sigmoid activation function, given by

$$
\sigma(x)=\frac{1}{1+e^{-x}} .
$$

4.3. Back-Propagation Neural Network (BPNN). Finally, BPNN is used to predict the power demands or outputs considering the nonlinear fitting ability of BPNN. Assuming that there are $N$ RBMs in $\mathrm{DBN}$, the final data feature extracted by DBN is $\left\{h_{1}^{N}, h_{2}^{N}, \ldots, h_{n}^{N}\right\}$. Considering that the output (denoted as $y$ ) of BPNN is the predicted results of resident loads, charging demands, and renewable outputs, therefore, the input and output of BPNN satisfy the following relationship:

$$
y=\sum_{q=1}^{A} \omega_{q} h_{q}^{N},
$$

where $\omega_{q}$ is the weight coefficient between the input neuron and output neuron in BPNN and $A$ is the number of neurons in the hidden layer.

The training method of BPNN is very mature. Literature [30] pointed out that BPNN mostly takes the minimum loss function as the objective function, but in order to avoid BPNN overfitting, a regular term can be added to the loss function:

$$
\begin{aligned}
J(\omega, h, y) & =J_{0}(\omega, h, y)+\alpha \Omega(\omega), \\
\Omega(\omega) & =\frac{1}{2}\|\omega\|_{2}^{2},
\end{aligned}
$$

where $\omega$ is the weight coefficient vector between the input neuron and the output neuron in BPNN, $h$ is the input feature vector, $\Omega$ is the penalty function, $J_{0}$ is the original loss function, and $J$ is the loss function considering the penalty function.

\section{Orderly Charging Scheduling Strategy for EVs}

Based on the forecasting model proposed in Section 4, the power forecasting values of residential distribution stations, centralized charging stations, and renewable plants in the distribution network can be obtained, which are denoted as $P_{r, t}^{R}, P_{u, t}^{C}$, and $P_{z, t}^{R E S}$ (where the subscripts " $r$ ", " $u$ ", and " $z$ " represent the numbers of residential distribution stations, centralized charging stations, and renewable plants, respectively; " $t$ " represents the time period taking value from $\{1,2, \ldots, 24\})$. Therefore, the orderly charging strategy proposed in this paper is divided into two stages:

(1) Setting the overall power supply plans for charging station: the control center in distribution network at cloud side determines the charging plan value of each charging station aiming at minimizing the operation cost of the distribution network according to the predicted results from the edge nodes.

(2) Setting the charging strategy for each EV:each charging station sets a personalized charging plan based on the actual charging needs of each EV, aiming at minimizing the deviation of the charging station's execution from the charging plan.

\subsection{Power Supply Plan for Charging Stations}

5.1.1. Objective Function. The control center in distribution network at cloud side sets the power supply plans for 
charging stations in each period of the next day according to the forecasting results collected from the edge nodes with the goal of minimizing the operation cost of the distribution network. The operating cost of the distribution network includes the electricity purchase cost from the superior grid and the income from renewable energy. Therefore, the objective function can be expressed as

$$
J_{1}=\sum_{t=1}^{24}\left(f_{t}^{\text {power }} P_{t}^{\text {power }}-f_{t}^{\mathrm{RES}} P_{t}^{\mathrm{RES}}\right),
$$

where $P_{t}^{\text {power }}$ is the power purchased by the distribution network to the superior grid; $f_{t}^{\mathrm{RES}}$ is the purchasing price; and $f_{t}^{\mathrm{RES}}$ is the on-grid electricity price of renewable energy. The relationships among $P_{t}^{\text {power }}$, charging station power supply plan (recorded as $\bar{P}_{u, t}^{C}$ ), $P_{r, t}^{\mathrm{R}}$, and $P_{z, t}^{\mathrm{RES}}$ satisfy equation (10):

$$
P_{t}^{\text {power }}=\sum_{r} P_{r, t}^{R}+\sum_{u} \bar{P}_{u, t}^{C}-\sum_{s} P_{s, t}^{\mathrm{RES}},
$$

where the charging station power supply plan is proportional to the charging station charging demand $P_{u, t}^{\mathrm{C}}$; i.e.,

$$
\bar{P}_{u, t}^{C}=\frac{P_{u, t}^{C}}{\sum_{u} P_{u, t}^{C}} \sum_{u} \bar{P}_{u, t}^{C} .
$$

Therefore, the power supply plan problem for charging stations can be described as determining the power supply plan $\bar{P}_{u, t}^{\mathrm{C}}$ for each charging station to minimize the operation cost of the distribution network.

5.1.2. Constraints. The following constraints need to be obeyed when formulating the charging plan for each charging station:

(1) The Restrictions on Renewable Energy Output. At any time $t$, the output of the renewable energy generators should not exceed the maximum allowed capacity of the distribution transformer, which can be described as

$$
0 \leq P_{s, t}^{\mathrm{RES}} \leq P^{\mathrm{cap}}
$$

(2) The Restrictions on Power Purchase in Distribution Network. At any time $t$, the power purchased by the distribution network to the superior gird should not exceed the maximum allowed capacity of the distribution transformer, which can be described as

$$
0 \leq P_{s, t}^{\text {power }} \leq P^{\text {cap }}
$$

(3) The Restrictions on Charging Demand. The power supply plan coordination of the charging station should not be less than the predicted value of charging demand at any time, which can be expressed as

$$
\bar{P}_{u, t}^{C} \geq P_{u, t}^{C}
$$

Therefore, the optimization problem of charging station power supply plan can be described as

O.P.: $\min J_{1}$

s.t. equations (12)-(14).

5.2. EV User Charging Plan Formulation. After each charging station obtains the charging plan curve, it needs to flexibly arrange the charging power and charging period of the EV users who have signed the contract with the charging station, so that the user's overall charging load curves change with the charging plan formulated by the control center in distribution network at cloud side. First of all, the assumptions are made for EV users and charging station operators as follows:

(1) EV users upload the EV statuses of charge, the rated battery capacity, the expected statuses of charge, and the travel plans.

(2) The distribution network operator reasonably arranges the charging time and charging power according to the travel needs of the EV user and reaches the charging statuses expected by the user when the users start to travel.

5.2.1. Objective Function. Disorderly charging behavior may cause the actual charging station load to be greater or less than the planned curve, which will result in additional purchase or load removal and create an increase in the operating cost of the distribution network. Therefore, the objective function is the deviation of the charging station's execution and the charging plan:

$$
J_{2}=\sum_{t=1}^{24}\left(\sum_{k=1}^{K} P_{k, u, t}^{\mathrm{ch}}-\bar{P}_{u, t}^{C}\right)^{2},
$$

where $P_{k, u, t}^{\mathrm{ch}}$ is the charging power of the $k$ th $\mathrm{EV}$ and $K$ is the number of EVs in the $u$ th charging station at the period $t$.

5.2.2. Constraints. For the charging station, the reasonable arrangement of the charging power and charging time of each EV needs to meet the following constraints:

(1) Charging Power Constraint. EV charging power at time $t$ has the upper and lower limits; i.e.,

$$
\underline{P}_{u, t}^{\mathrm{ch}} \leq P_{k, u, t}^{\mathrm{ch}} \leq \bar{P}_{u, t}^{\mathrm{ch}},
$$

where $\underline{P}_{u, t}^{\text {ch }}$ and $\bar{P}_{u, t}^{\text {ch }}$ are the minimum and maximum charging power of the $\mathrm{EV}$, respectively.

(2) State of Charge (SoC) Constraint. The SoC level of EV battery guarantees the user's maximum travel distance. In addition, to avoid overcharging of the EV battery that affects the service life, the SoC is not allowed to exceed $100 \%$ when participating in the charging scheduling process. Secondly, the charging station needs to ensure that the EV reaches the setting SoC level before the user's 
expected travel starting time. Finally, after reaching the expected SoC preset by the EV user, the charging station will immediately stop charging the EV. Therefore, the constraints are shown as

$$
\begin{gathered}
S o C_{k, u, t}^{0}+\sum_{t=t_{k, u}^{0}}^{t_{k, u}^{\text {setting }}} \frac{\delta_{k, u, t} P_{k, u, t}^{\mathrm{ch}} \Delta T_{k, u, t}}{C_{k, u}^{\text {rated }}} \times 100 \% \\
\leq \min \left\{S o C_{k, u, t}^{\text {setting }}, 100 \%\right\}, \\
\underset{t}{\arg \max }\left\{\Delta T_{k, u, t}\right\} \leq t_{k, u}^{\text {setting }},
\end{gathered}
$$

where $\mathrm{SoC}_{k, u, t}^{0}$ and $\mathrm{SoC}_{k, u, t}^{\text {setting }}$ are the starting SoC and expected SoC of the $k$ th EV, respectively; $\delta_{k, u, t}$ are the $0-1$ decision variables which determine whether to charge the $k$ th EV at time $t ; \Delta T_{k, u, t}$ is the charging time of the $k$ th EV in the time period $t ; C_{k, u}^{r a t e d}$ is the rated capacity of the EV battery; $t_{k, u}^{0}$ is the initial charging time of the $k$ th EV; $t_{k, u}^{\text {setting }}$ is the expected charging end time set by the user of the $k$ th EV; $\operatorname{argmax}(g(x))$ returns the variable $x$ that the function $g(x)$ takes the maximum value.

(3) Constraint on the Number of Charging Times. Frequent charging of the EV battery within a day is also harmful to its service life. Therefore, the number of charging times of any $\mathrm{EV}$ in a day has the following constraint:

$$
\operatorname{Num}\left(\delta_{k, u, t}=1\right) \leq M \text {. }
$$

Therefore, the EV charging scheduling strategy optimization problem at the charging station level can be described as

O.P.: $\min J_{2}$

s.t. equations (16)-(19).

Note that there exists a multiplicative relationship between the variables $\Delta T_{k, u, t}$ and $\delta_{k, u, t}$; therefore, the optimal charging scheduling scheme is a nonlinear integer programming problem. The method proposed in [24] is used here to obtain the final results.

\section{Simulations and Results}

A $10.5 \mathrm{kv}$ distribution network in a province in North China is taken as an example in this section. The distribution network has three branches. As shown in Figure 3, each node has edge computing capabilities. There are distributed renewable energy power stations based on wind turbines (WTs) and photovoltaic panels (PVs) at the ends of the upper and lower branches. The installed capacity of WTs is $1.5 \mathrm{MVA}$ and the capacity of PVs is 1.0 MVA. Nodes 5 and 11 are EV charging stations with the capacity of 2.0 MVA.

6.1. Experimental Parameters. All algorithms are run in the same software and hardware environment. The experimental equipment is Windows10 64-bit operating system, the processor is Intel i7-7700 CPU @ $3.60 \mathrm{~Hz}$, the running

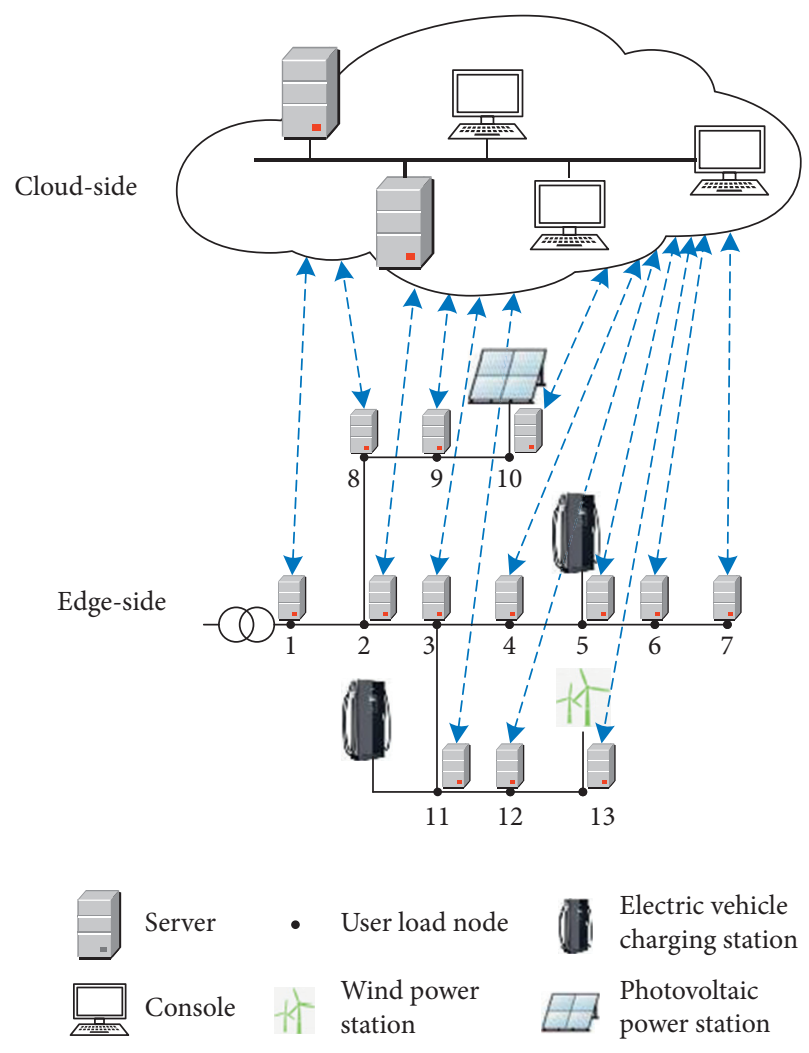

Figure 3: The distribution network branches.

memory is $8 \mathrm{~GB}$, the software platform is Python 3.7.3, and the Keras deep learning framework is adopted. The input variables and output variables of CNN-DBN are shown in Table 1, and the training parameters of CNN-DBN are shown in Table 2.

\subsection{Analysis of Experimental Results}

6.2.1. Load Forecasting Results. In this case, all edge nodes have computing ability and can predict residential loads, charging demands, and renewable outputs. The long shortterm memory (LSTM) network model proposed in [20] and the linear regression (LR) method proposed in [10] are selected as comparison algorithms. The comparison of the forecasting results and the real values is shown in Figure 4. It should be pointed out that the curves in Figure 4 are the sum of the results obtained from the edge computing nodes of the residential load areas.

As shown in Figure 5, for the traditional load forecasting, the results with proposed CNN-DBN method are more consistent with the real value. The average absolute errors of the three algorithms are $0.064 \mathrm{MW}, 0.092 \mathrm{MW}$, and $0.174 \mathrm{MW}$, respectively. Compared with the other two algorithms, the average absolute errors of the proposed CNNDBN method forecasting results are reduced by $30.34 \%$ and $62.85 \%$. From the box plot and the fitted normal distribution, it can be seen that the forecasting error distribution of the proposed CNN-DBN method is more concentrated and the fluctuations are also smaller. In addition, in terms of 
TABLE 1: Input and output variables of CNN-DBN.

\begin{tabular}{lcc}
\hline Variables & Symbols & Variable name \\
\hline & $x_{1}$ & Pressure \\
Environment variables (inputs) & $x_{2}$ & Temature \\
& $x_{3}$ & Relative humidity \\
& $x_{4}$ & Wind speed \\
& $x_{5}$ & Solar radiation intensity \\
Temporal variables (inputs) & $x_{6}$ & Latitude \\
& $x_{7}$ & Month \\
& $x_{8}$ & Week \\
Outputs & $x_{9}$ & Holiday \\
\hline
\end{tabular}

local maximum errors, the maximum absolute error of DBN is only $0.128 \mathrm{MW}$ for the residential load while the maximum absolute errors of the other two algorithms are $0.199 \mathrm{MW}$ and $0.320 \mathrm{MW}$, respectively. The numerical results show that, with the proposed CNN-DBN method, the forecasting accuracy within a day can be maintained at a high level.

As shown in Figures 6-8, for the EV charging demands, the forecasting results of the proposed CNN-DBN algorithm also have better forecasting results. Specially, for charging station 1, the average absolute errors of the three algorithms are $0.016 \mathrm{MW}, 0.024 \mathrm{MW}$, and $0.051 \mathrm{MW}$, respectively. Compared with the other two algorithms, the average absolute error of the CNN-DBN method is reduced by $32.67 \%$ and $69.15 \%$, respectively. For charging station 2, the average absolute errors of the three algorithms are $0.016 \mathrm{MW}$, $0.026 \mathrm{MW}$, and $0.028 \mathrm{MW}$. Compared with the other two algorithms, the average absolute errors of CNN-DBN method are reduced by $38.92 \%$ and $42.68 \%$, respectively. It can also be seen that the differences between the upper and lower quartiles of forecasting results with $\mathrm{CNN}-\mathrm{DBN}$ are both less than $0.02 \mathrm{MW}$ in charging station 1 and charging station 2, while the quartile differences of other forecasting methods are above $0.023 \mathrm{MW}$. Therefore, the distribution of forecasting errors with CNN-DBN method is more concentrated, and the forecasting accuracy fluctuates less. In addition, some results about the local maximum error are analyzed. For charging station 1, the maximum absolute error of CNN-DBN is only 0.031 MW, while the other two algorithms are $0.050 \mathrm{MW}$ and $0.125 \mathrm{MW}$, respectively; for charging station 2, the maximum absolute error of $\mathrm{CNN}$ DBN is only $0.029 \mathrm{MW}$, while the maximum absolute errors of the other two algorithms are $0.050 \mathrm{MW}$ and $0.061 \mathrm{MW}$, respectively. Therefore, the algorithm proposed in this paper is obviously better.

In summary, whether it is for residential load or EV demands with large demand fluctuations, the proposed CNN-DBN based forecasting method has obvious advantages compared with the traditional LR and LSTM methods. Therefore, the proposed CNN-DBN method can give full
TABLE 2: Training parameters.

\begin{tabular}{lc}
\hline Parameter & Value \\
\hline Convolution kernel size of CNN & $3 \times 3$ \\
Convolution level of CNN & 6 \\
Number of RBMs in DBN & 3 \\
Training times & 200 \\
Training sample size of CNN & 5000 \\
Learning rate of RBM & 0.001 \\
Training sample size of RBM & 5000 \\
Learning rate of BPNN & 0.001 \\
Training sample size of BPNN & 5000 \\
\hline
\end{tabular}

play to the computing capacities of the edge computing nodes to obtain more accurate forecasting results which can be used for the subsequent orderly scheduling of EVs.

6.2.2. Renewable Output Forecasting. Similarly, the outputs of renewable plants (i.e., the wind and PV plants) are forecasted at the edge computing nodes. The forecasting results are shown in Figures 9 and 10. The forecasting conducted by the proposed CNN-DBN algorithm for renewable plants is analyzed. It can be seen that the CNN-DBN algorithm has a smaller deviation from the true value and is significantly better than the other two algorithms. The absolute error box-plot is shown in Figure 11.

For the forecasting results of photovoltaic power generation, the average absolute errors of the three algorithms are $0.006 \mathrm{MW}, 0.014 \mathrm{MW}$, and $0.019 \mathrm{MW}$, respectively. Compared with the other two algorithms, the average absolute errors of the proposed CNN-DBN method are reduced by $55.548 \%$ and $66.862 \%$, respectively. For the forecasting results of wind power generation, the average absolute errors of the three algorithms are $0.023 \mathrm{MW}, 0.043 \mathrm{MW}$, and $0.056 \mathrm{MW}$, respectively. Compared with the other two algorithms, the average absolute error of the proposed CNN-DBN method is reduced by $45.73 \%$ and $58.58 \%$, respectively. From the box-plot and the fitted normal distribution, it can be seen that whether it is between the upper and lower quartiles or 


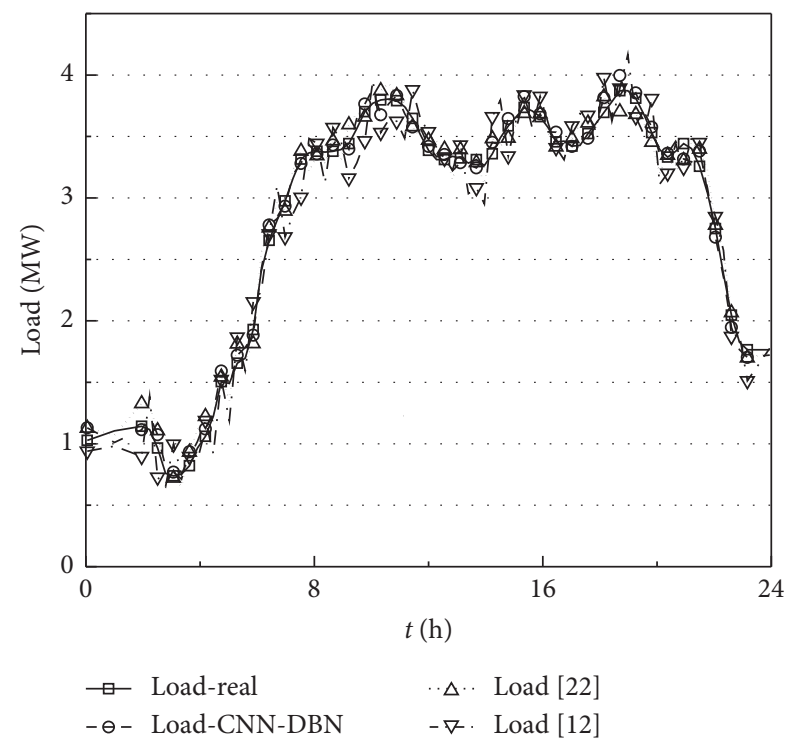

Figure 4: Load forecasting results of residential loads.

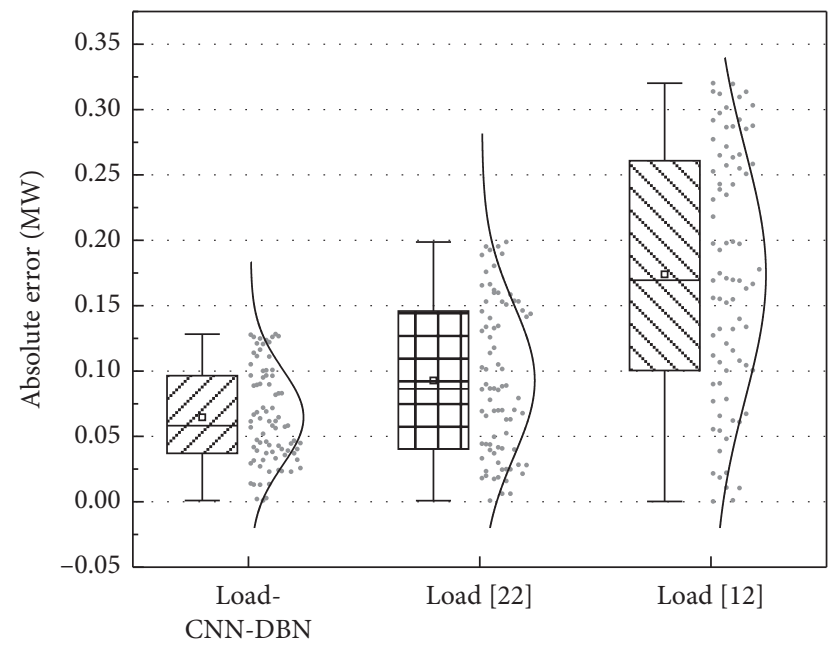

FIgURE 5: Error analysis of residential load forecasting results.

the overall error distribution, the forecasting error of the proposed CNN-DBN method is more concentrated and the fluctuations are smaller, and the forecasting results are more stable. In addition, in terms of local maximum errors, for photovoltaic power generation, the maximum absolute error of the proposed CNN-DBN method is $0.039 \mathrm{MW}$, while the maximum absolute errors of the other two algorithms are $0.058 \mathrm{MW}$ and $0.090 \mathrm{MW}$, respectively. For wind power generation, the maximum absolute errors of the other two algorithms are $0.098 \mathrm{MW}$ and $0.115 \mathrm{MW}$, while the error of CNN-DBN method is $0.050 \mathrm{MW}$, which are significantly smaller than the other two algorithms.

In summary, in terms of average error, global error distribution, and local maximum error, the forecasting effect of the algorithm proposed in this paper is significantly better than the traditional LR and LSTM methods.

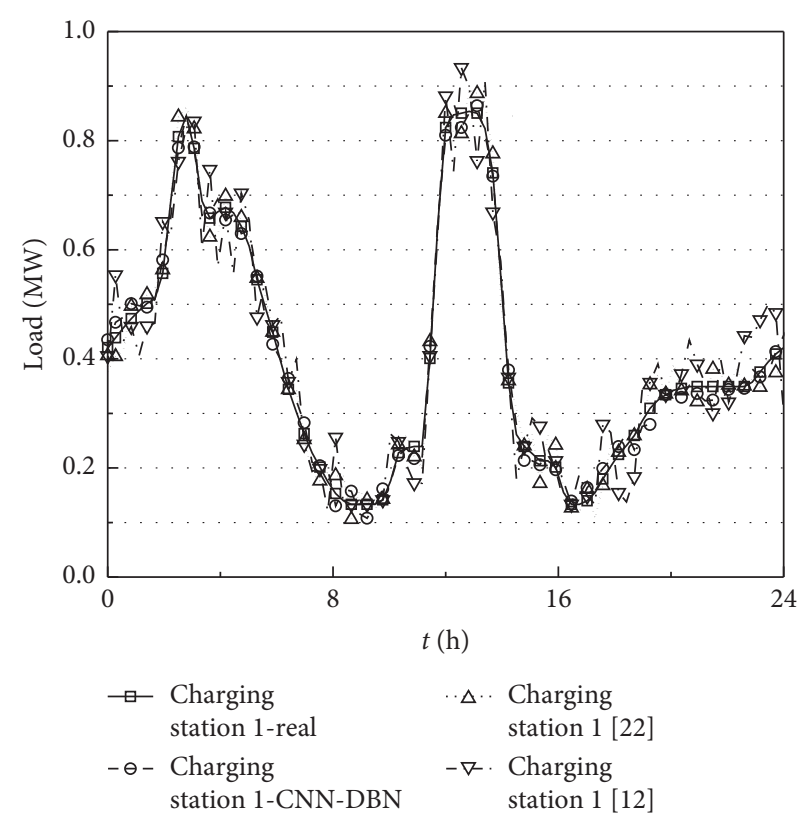

FIGURE 6: Forecasting results of charging demands in charging station 1.

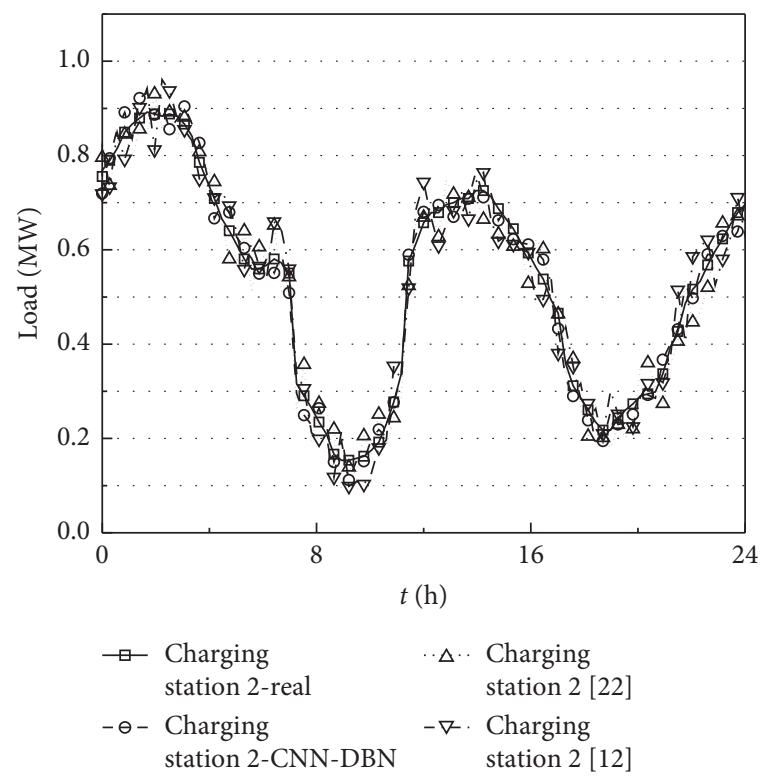

Figure 7: Forecasting results of charging demands in charging station 2 .

6.2.3. Orderly Scheduling for EVs. The charging load curves after scheduling in charging stations 1 and 2 are shown in Figures 2 and 3 , respectively. The disordered charging method and the orderly scheduling method proposed in [22] are adopted as the comparison algorithm. From Figures 12 and 13, it can be seen that for the two EV charging stations, after the orderly scheduling with the goal of minimizing the operation cost of the distribution network, the actual charging load curve fits well with the planned curve, and the load follows the input changes well. This method has obvious advantages compared with the disordered charging method 


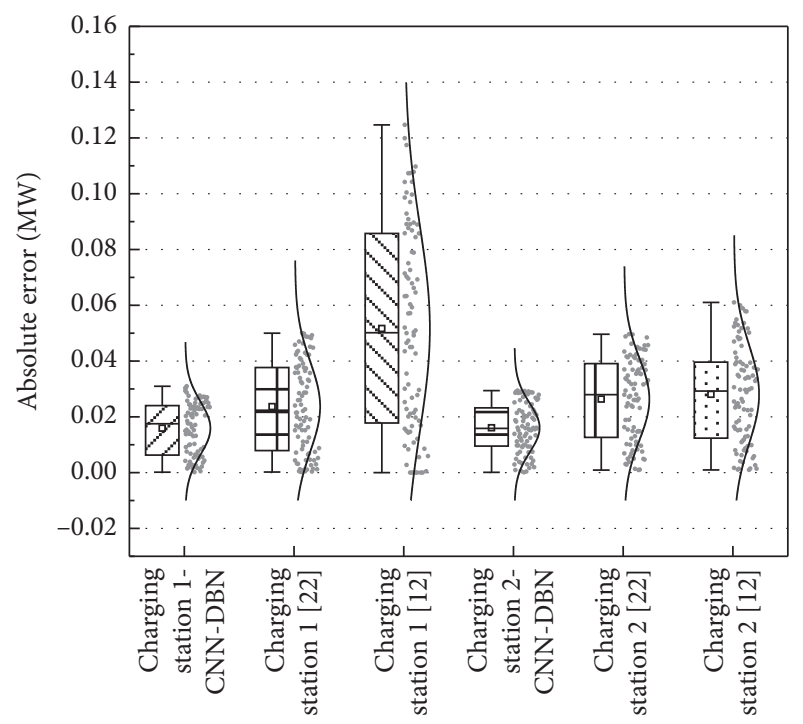

FIGURE 8: Error analysis of charging demands in charging stations 1 and 2 .

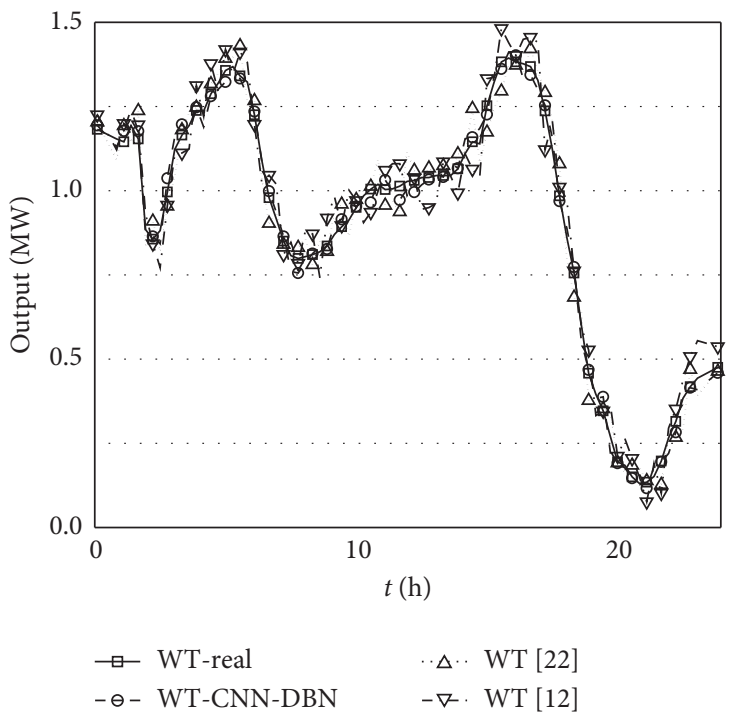

FIGURE 9: Forecasting results of wind plant.

and the method proposed in [22]. For charging station 1, the maximum absolute error of the orderly scheduling result is only $0.033 \mathrm{MW}$ compared with the planned curve, which occurs at about 0:00 at night. The planned output curve value at this time is too high, and the scheduling is not flexible at this time period, which makes the charging load lower than the planned load in this situation. However, the charging load curve in the day can basically follow the planned value completely, and it can still basically follow the load when the planned load changes rapidly. For example, if the plan meets the sudden drop at around 6:00 in the morning, the orderly dispatch method in this paper can still basically follow the planned load curve, but the maximum absolute error of the method used in [22] reaches $0.095 \mathrm{MW}$. For charging station 2 , the orderly scheduling charging load curve proposed in this paper can still follow the changes, and the effect is better

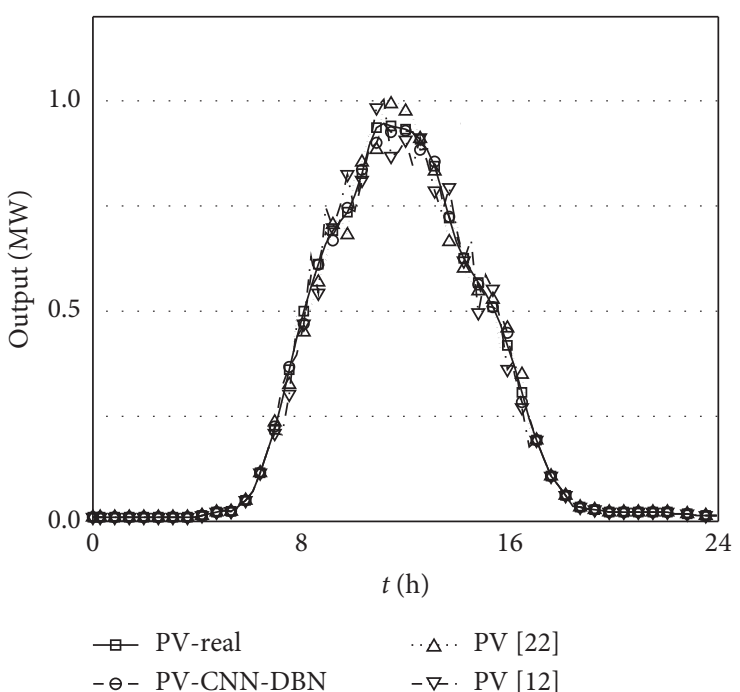

FIGURE 10: Forecasting results of PV plant.

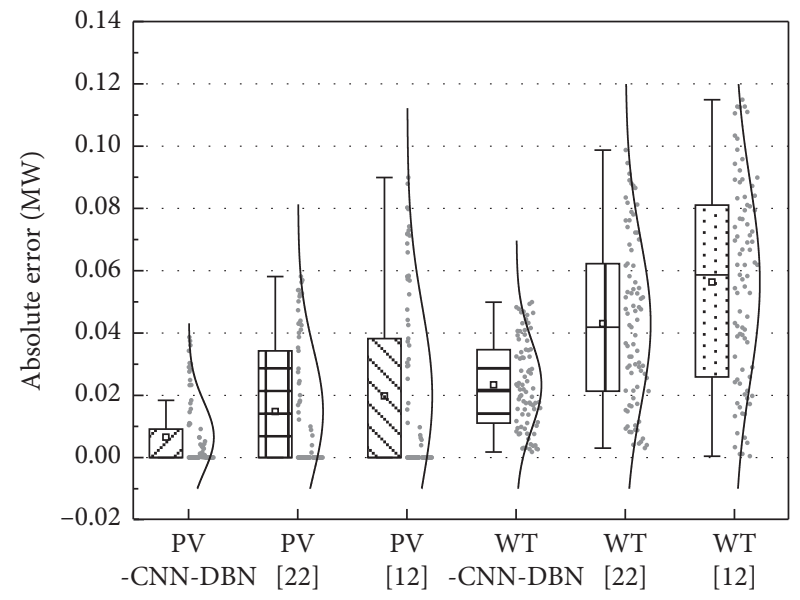

FIGURE 11: Error analysis of the renewable output forecasting.

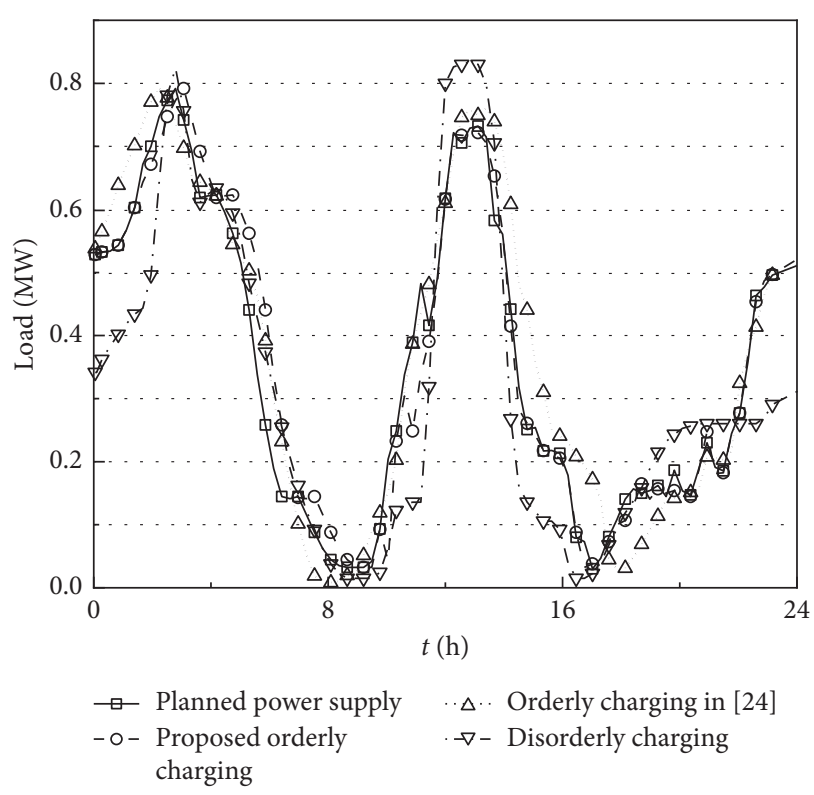

FIGURE 12: Orderly scheduling results of charging station 1. 


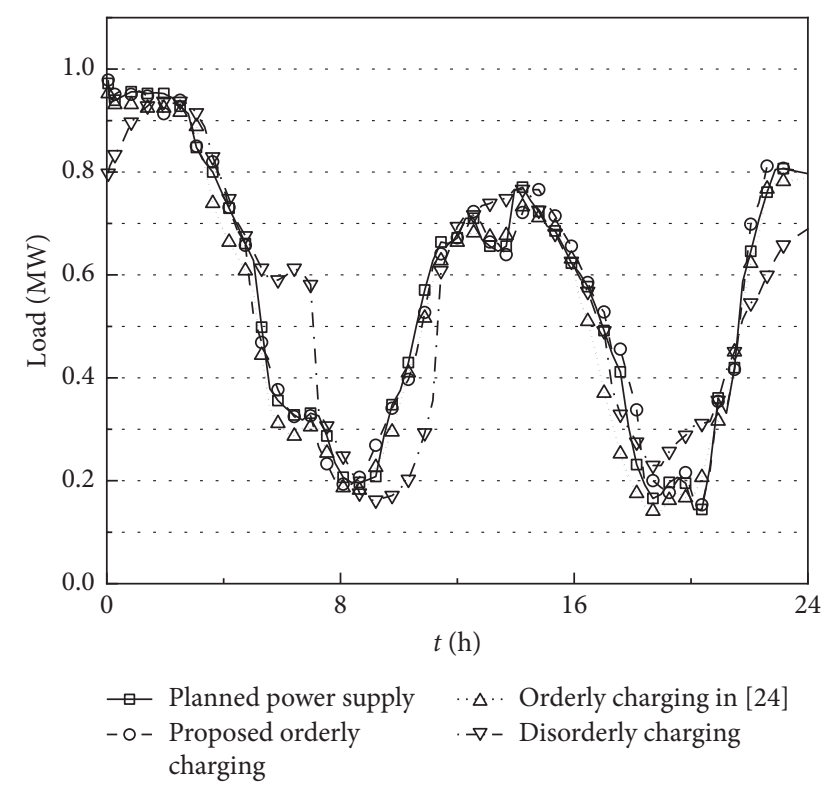

FIgURE 13: Orderly scheduling results of charging station 2.

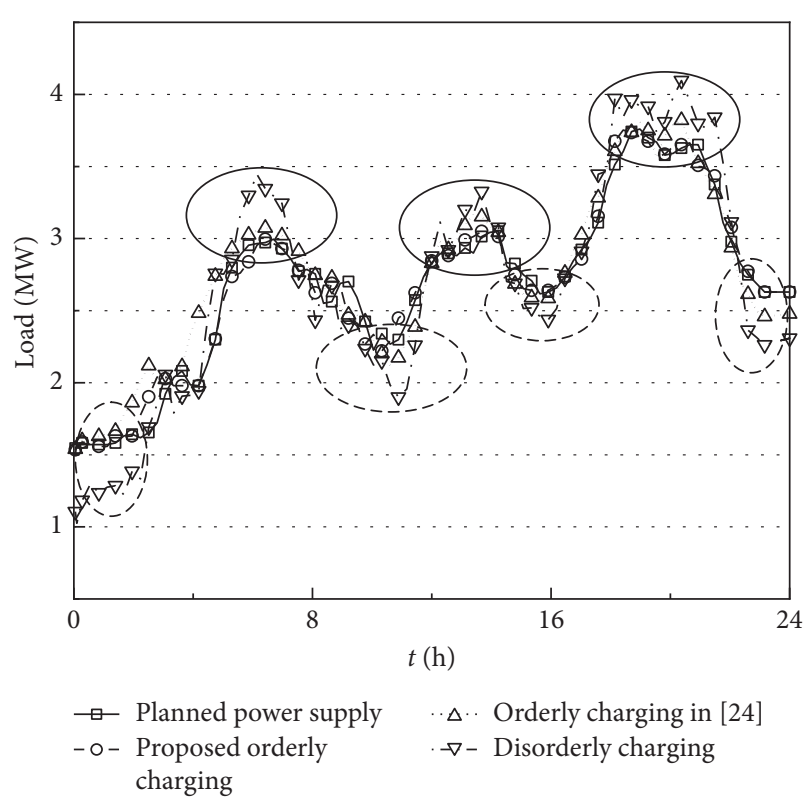

FIgURE 14: Total load curve optimization results.

than that of the method used in [22], and the maximum absolute error is only $0.025 \mathrm{MW}$ compared to the planned curve. In addition, for charging station 1 , the root mean square error between actual charging load curve and planned load curve is $0.02 \mathrm{MW}$. Compared with the scheduling method proposed in [22] and the disorderly charging method, the root mean square error between the two curve is reduced by $25.35 \%$ and $54.51 \%$, respectively. Similar conclusions can be drawn in charging station 2 .

Figure 14 shows the total load curve after conducting the orderly scheduling for charging stations 1 and 2 . It can be seen that the total load curve of EVs has been optimized after conducting the orderly scheduling. Partially, the total load of each wave peak has been weakened. In order to supplement the weakened controllable load, the total load of each trough is filled. As shown in the internal load curve of the solid line ellipse in Figure 14, the total load of each wave peak is weakened, and the total load of each valley inside the dotted ellipse is filled. The average orderly scheduled load is almost the same as the planned average load. The daily average load of planned dispatch is $2.682 \mathrm{MW}$. After orderly scheduling, the average daily load becomes $2.683 \mathrm{MW}$, so the difference is only $0.001 \mathrm{MW}$. In terms of load fluctuations, after using the scheduling method proposed in this paper, the maximum peak-to-valley difference of the load fluctuation within a day is $2.261 \mathrm{MW}$, which is $30.13 \%$ less than the result of disordered charging and $3.16 \%$ less than the result of the method used in [22]; the overall deviation is $0.667 \mathrm{MW}$, which is reduced by $0.136 \mathrm{MW}$ compared with disordered charging and by $0.026 \mathrm{MW}$ compared with the method used in [22]. And the curve becomes smoother after orderly scheduling.

In summary, based on the goal of minimizing the distribution network operation cost, the EV scheduling method proposed in this paper can not only realize the effective follow-up of the planned load curve, but also optimize the total load curve of the overall distribution network. Using this strategy, the daily load curve of the distribution network is smoothed, and the adverse impact of load fluctuations on the distribution network are reduced at the same time.

\section{Conclusion}

Aiming at reducing the influence of disorderly charging behaviors of large-scale EVs on the safe operation of distribution networks, this paper proposes an orderly charging scheduling strategy for EVs based on cloud-side collaboration and deep learning method. Based on the forecasting of residential loads, charging demands, and the renewable outputs at the edge computing nodes, the control center in distribution network at cloud side formulates a power supply plan for each charging station. Then each charging station reasonably arranges the charging power and charging time period of each EV according to the power supply plan curve. Case studies show that, compared with the conventional algorithms, the proposed algorithm has better performance in improving the power forecasting accuracy and suppressing the load peak-to-valley differences. In the future, we will further study the method to improve the forecasting accuracy based on deep learning and EV charging scheduling strategy that considers more actual operation constraints of the distribution network.

\section{Data Availability}

The data used to support the findings of this study are available from the corresponding author upon request.

\section{Conflicts of Interest}

The authors declare that they have no conflicts of interest regarding the publication of this paper. 


\section{Acknowledgments}

This work was supported by the National Key R\&D Program of China (2016YFB0900600).

\section{References}

[1] S. Wang, S. Bi, and Y. Zhang, "A reinforcement learning approach for EV charging station dynamic pricing and scheduling control," 2018 IEEE Power \& Energy Society General Meeting (PESGM), 2018.

[2] K. Chaudhari, A. Ukil, K. N. Kumar, U. Manandhar, and S. K. Kollimalla, "Hybrid optimization for economic deployment of ESS in PV-integrated EV charging stations," IEEE Transactions on Industrial Informatics, vol. 14, no. 1, pp. 106-116, 2018.

[3] T. Alicia, A. Jos, and T. Sebastian, "Joint routing and scheduling for electric vehicles in smart grids with V2G," Energy, vol. 175, pp. 113-122, 2019.

[4] T. Yang, Y. Zhang, Z. Wang et al., "Secondary frequency stochastic optimal control in independent microgrids with virtual synchronous generator-controlled energy storage systems," Energies, vol. 11, pp. 1-14, 2018.

[5] J. Ke, X. Cen, H. Yang, X. Chen, and J. Ye, "Modelling drivers' working and recharging schedules in a ride-sourcing market with electric vehicles and gasoline vehicles," Transportation Research Part E: Logistics and Transportation Review, vol. 125, pp. 160-180, 2019.

[6] Ş. İbrahim, C. Alper, K. Ayse et al., "User-comfort oriented optimal bidding strategy of an electric vehicle aggregator in day-ahead and reserve markets," International Journal of Electrical Power and Energy Systems, vol. 122, pp. 1-15, 2020.

[7] H. Hui, X. Meng, X. Yan et al., "Multi-objective economic dispatch of a microgrid considering electric vehicle and transferable load," Applied Energy, vol. 262, pp. 1-10, 2020.

[8] Y. Tao, M. Huang, Y. Chen et al., "Orderly charging strategy of battery electric vehicle driven by real-world driving data," Energy, vol. 193, pp. 1-9, 2020.

[9] H. Jiang, S. Ning, and Q. Ge, "Multi-objective optimal dispatching of microgrid with large-scale electric vehicles," IEEE Access, vol. 7, pp. 145880-145888, 2019.

[10] G. Dudek, "Pattern-based local linear regression models for short-term load forecasting," Electric Power Systems Research, vol. 130, pp. 139-147, 2016.

[11] Q. Duan, J. Liu, and D. Zhao, "A fast algorithm for short term electric load forecasting by a hidden semi-markov process," Journal of Statistical Computation and Simulation, vol. 89, no. 5, pp. 831-843, 2019.

[12] Z.-C. Yang, "Electric load movement evaluation and forecasting based on the Fourier-series model extend in the leastsquares sense," Journal of Control, Automation and Electrical Systems, vol. 26, no. 4, pp. 430-440, 2015.

[13] N. Huang, G. Lu, and D. Xu, "A permutation importancebased feature selection method for short-term electricity load forecasting using random forest," Energies, vol. 9, pp. 1-24, 2016.

[14] A. Lahouar and J. Ben Hadj Slama, "Day-ahead load forecast using random forest and expert input selection," Energy Conversion and Management, vol. 103, pp. 1040-1051, 2015.

[15] Y. Hu, J. Li, M. Hong et al., "Short term electric load forecasting model and its verification for process industrial enterprises based on hybrid GA-PSO-BPNN algorithm-A case study of papermaking process," Energy, vol. 170, pp. 12151227, 2019.
[16] M. Barman, N. B. Dev Choudhury, and S. Sutradhar, "A regional hybrid Goa-SVM model based on similar day approach for short-term load forecasting in Assam, India," Energy, vol. 145, pp. 710-720, 2018.

[17] E. Choi, S. Cho, and D. Kim, "Power demand forecasting using long short-term memory (LSTM) deep-learning model for monitoring energy sustainability," Sustainability, vol. 12, pp. 1-14, 2020.

[18] L. Wu, C. Kong, X. Hao, and W. Chen, "A short-term load forecasting method based on GRU-CNN hybrid neural network model," Mathematical Problems in Engineering, vol. 2020, Article ID 1428104, 10 pages, 2020.

[19] H. J. Sadaei, P. C. de Lima e Silva, F. G. Guimaraes, and M. H. Lee, "Short-term load forecasting by using a combined method of convolutional neural networks and fuzzy time series," Energy, vol. 175, pp. 365-377, 2019.

[20] A. Santra and J. Lin, "Integrating long short-term memory and genetic algorithm for short-term load forecasting," Energies, vol. 12, pp. 1-11, 2019.

[21] M. Barman and N. Choudhury, "A similarity based hybrid GWO-SVM method of power system load forecasting for regional special event days in anomalous load situations in Assam, India," Sustainable Cities and Society, vol. 61, pp. 1-10, 2020.

[22] X. Kong, L. Bai, Q. Hu, F. Li, and C. Wang, "Day-ahead optimal scheduling method for grid-connected microgrid based on energy storage control strategy," Journal of Modern Power Systems and Clean Energy, vol. 4, no. 4, pp. 648-658, 2016.

[23] B. Jha, A. Singh, A. Kumar et al., "Day ahead scheduling of PHEVs and D-BESSs in presence of DGs in distribution system," IET Electrical Systems in Transportation, vol. 10, pp. 170-184, 2020.

[24] S. Mehdi, G. Reza, and M. Moein, "Day-ahead resource scheduling in distribution networks with presence of electric vehicles and distributed generation units," Electric Power Components and Systems, vol. 47, pp. 1450-1463, 2019.

[25] S. Dong, S. Xu, J. Liang, Z. Li, X. Li, and Z. Zhang, "Optimal day-ahead scheduling of smart microgrid considering powerto-gas technology," Advances in Intelligent Systems and Computing, vol. 752, pp. 847-853, 2018.

[26] B. Rasouli, M. Salehpour, J. Wang et al., "Optimal day-ahead scheduling of a smart micro-grid via a probabilistic model for considering the uncertainty of electric vehicles' load," Applied Sciences, vol. 9, pp. 1-23, 2019.

[27] H. Yuan, G. Wei, L. Zhu et al., "Optimal scheduling for microgrid considering EV charging-swapping-storage integrated station," IET Generation, Transmission and Distribution, vol. 14, no. 6, pp. 1127-1137, 2020.

[28] Y. Zheng, Y. Song, D. J. Hill, and K. Meng, “Online distributed MPC-based optimal scheduling for EV charging stations in distribution systems," IEEE Transactions on Industrial Informatics, vol. 15, no. 2, pp. 638-649, 2019.

[29] W. Tang and Y. J. Zhang, "A model predictive control approach for low-complexity electric vehicle charging scheduling: optimality and scalability," IEEE Transactions on Power Systems, vol. 32, no. 2, pp. 1050-1063, 2017.

[30] H. K. Jia, L. D. Yu, and Y. Z. Jiang, "Compensation of rotary encoders using fourier expansion-back propagation neural network optimized by genetic algorithm," Sensors, vol. 20, no. 9, p. 2603, 2020. 